\title{
WHITE NOISE GENERALIZATION OF THE CLARK-OCONE FORMULA UNDER CHANGE OF MEASURE
}

\author{
YELIZ YOLCU OKUR \\ Centre of Mathematics for Applications \\ Institute of Mathematics, University of Oslo \\ N-0316 Oslo, Norway \\ E-mail: yelizy@math.uio.no
}

\begin{abstract}
We proved white noise generalization of the Clark-Ocone formula under change of measure by using white noise analysis and Malliavin calculus. Let $W(t)$ be a Brownian motion on the filtered white noise probability space $\left(\Omega, \mathcal{B},\left\{\mathcal{F}_{t}\right\}_{t \geq 0}, P\right)$ and let $\hat{W}(t)$ be defined as $d \hat{W}(t)=$ $u(t)+d W(t)$, where $u(t)$ is an $\mathcal{F}_{t}$-measurable process satisfying certain conditions. Let $\mathrm{Q}$ be the probability measure equivalent $\mathrm{P}$ such that $\hat{W}(t)$ is a Brownian motion with respect to $\mathrm{Q}$, in virtue of the Girsanov theorem. In this paper, it is shown that for any random variable $F \in L^{2}(P)$

$$
F(\omega)=E_{Q}[F]+\int_{0}^{T} E_{Q}\left[\left(D_{t} F-F \int_{t}^{T} D_{t} u(s) d \hat{W}(s)\right) \mid \mathcal{F}_{t}\right] d \hat{W}(t),
$$

where $E_{Q}$ is the expectation under $Q$ and $D_{t} F(\omega)$ is the (Hida) Malliavin derivative. The important point to note here is in this settlement $F$ need not belong to stochastic Sobolev space, $\mathbb{D}_{1,2}$ which is subset of $L^{2}(P)$. This makes this formula more useful in applications to finance. For example, the replicating portfolio for a digital option, whose payoff function $\chi_{[K, \infty)} W(T) \notin \mathbb{D}_{1,2}$, is calculated by using this generalized Clark-Ocone formula under change of measure.
\end{abstract}

1. Introduction. Gaussian white noise theory was first introduced by Hida [6]. Afterwards, it is developed by him and other researchers that becomes a powerful tool in mathematical physics (see [7] and the references therein). After that H. Holden et al [8] emphasized this theory with stochastic partial differential equations (SPDEs) driven by Brownian motion. The first contribution to white noise theory to finance comes from the

2000 Mathematics Subject Classification: Primary 46C20; Secondary 32G81.

Key words and phrases: Clark-Ocone formula, Malliavin calculus, (Hida) Malliavin derivative, Change of measure.

The author wishes to express her thanks to Prof. Bernt Øksendal for suggesting the problem and for several helpful comments.

The paper is in final form and no version of it will be published elsewhere. 
joint work of K. Aase et al to prove the generalization of Clark-Ocone formula [1]. By this theorem it is a natural and intrinsic way of computing the replicating portfolio of call option in Black \& Scholes type market. They proved that

$$
F(\omega)=E[F]+\int_{0}^{T} E\left[D_{t} F \mid \mathcal{F}_{t}\right] \diamond \dot{W}(t) d t .
$$

Here $E[F]$ denotes the generalized expectation, $D_{t} F(\omega)=\frac{d F}{d \omega}$ is the (generalized) Malliavin derivative, $\diamond$ is the Wick product and $\dot{W}(t)$ is 1-dimensional Gaussian white noise. This formula holds for all $F \in \mathcal{G}^{*} \supset L^{2}(P)$, where $\mathcal{G}^{*}$ is a space of stochastic distributions and $P$ is the white noise probability measure.

In particular, if $F \in L^{2}(P)$ then equation (1) turns out to be

$$
F(\omega)=E[F]+\int_{0}^{T} E\left[D_{t} F \mid \mathcal{F}_{t}\right] d W(t)
$$

where $W$ is the 1-dimensional Wiener process (Brownian motion) and $D_{t} F \in \mathcal{G}^{*}$.

Actually, the original Clark-Ocone formula was proved by Ocone in 1984 to give an explicit representation to the integrand in Itô integral representation theorem in the context of analysis on the Wiener space $\Omega=C_{0}([0, T])$, the space of real continuous functions on $[0, T]$ starting at zero $[13]$. He proved that

$$
F(\omega)=E[F]+\int_{0}^{T} E\left[D_{t} F \mid \mathcal{F}_{t}\right] d B(t),
$$

where $D_{t}$ is the Malliavin derivative and $B(t)=B(t, \omega)$ is one dimensional Brownian motion on the Wiener space. In case of changing measure by Girsanov theorem, Karatzas \& Ocone 1991 [10] argued that under some assumptions the Clark-Ocone formula under change of measure is

$$
F(\omega)=E_{Q}[F]+\int_{0}^{T} E_{Q}\left[\left(D_{t} F-F \int_{t}^{T} D_{t} u(s) d \hat{B}(s)\right) \mid \mathcal{F}_{t}\right] d \hat{B}(t),
$$

where $D_{t}$ is the Malliavin derivative defined on Wiener space and $\hat{B}(t)$ is one dimensional Brownian motion under probability measure $Q$. However, the main drawback with this setting is that the Malliavin derivative only exists for $F \in \mathbb{D}_{1,2}$. The problem is that this settlement excludes many interesting applications in finance, such as digital option. The purpose of this paper is to represent a new proof of the Clark-Ocone formula under change of measure in the white noise setting.

The first three sections of this paper constitute sufficient preparation to prove the Clark-Ocone formula under change of measure for $F \in L^{2}(P)$. In the section 2 and 3 we summarize without proofs the relevant material on white noise analysis and special functions respectively. Many versions of those results have already been provided and known by many theorists but we think that it is better to have a unified approach based on white noise theory. In section 4, we introduce the notion of chaos expansion and define two different kinds of chaos expansions. Section 5 contains a brief summary of Hida stochastic test function, distribution space and the Malliavin derivative on this space. In section 6 we develop the theory of Clark-Ocone formula under change of measure on the 
analysis of white noise settlement. In the last section, we apply our result to compute the replicating portfolio for a digital option in a Black-Scholes type market.

2.White noise analysis. In this section we will set up notation and terminology in Gaussian white noise theory.

Let $S=S(\mathbb{R})$ be the Schwartz space of rapidly decreasing smooth functions $\phi \in C^{\infty}(\mathbb{R})$ such that

$$
\|\phi\|_{k, n}=\sup _{x \in \mathbb{R}}\left|\phi^{(j)}(x) x^{n}\right|<\infty \text { for all } j \leq k, n \leq N .
$$

The space $S(\mathbb{R})$ equipped with the family of seminorms \|\|$_{k, N}$ constitutes a Frechet space. The dual of $S(\mathbb{R})$ is denoted by $S^{\prime}(\mathbb{R})$ and called the space of tempered distributions equipped with the weak-star topology. If $\omega \in S^{\prime}(\mathbb{R})$ and $\phi \in S(\mathbb{R})$ we set

$$
\omega(\phi)=<\omega, \phi>
$$

which denotes the action of the linear functional $\omega$ on the test function $\phi$.

\section{THEOREM 1. (Bochner-Minlos)}

Let $g: S(\mathbb{R}) \rightarrow \mathbb{R}$ be a given function. Then there exists a probability measure $P$ on $\Omega:=S^{\prime}(\mathbb{R})$ s.t.

$$
\int_{S^{\prime}(\mathbb{R})} e^{i<\omega, \phi>} d P(\omega)=g(\phi) ; \phi \in S(\mathbb{R})
$$

if and only if

- $g(0)=1$

- $g$ is positive definite

- $g$ is continuous in Frechet topology.

Note that if we take $g(\phi)=e^{-\frac{1}{2}\|\phi\|_{L^{2}(\mathbb{R})}^{2}}$ which satisfies the above conditions then by Bochner-Minlos theorem there exists probability measure $P$ on $\Omega=S^{\prime}(\mathbb{R})$ such as the following equality holds:

$$
\int_{S^{\prime}(\mathbb{R})} e^{i<\omega, \phi>} d P(\omega)=e^{-\frac{1}{2}\|\phi\|_{L^{2}(\mathbb{R})}^{2}} .
$$

Moreover, this probability measure is called white noise probability measure and it is defined on the set $\mathcal{B}$ of Borel sets of $\Omega$. The triple $\left(\Omega=S^{\prime}(\mathbb{R}), \mathcal{B}, P\right)$ is called the white noise probability space. From the settlement (4) and by using the Taylor expansion we can easily prove that

$$
\begin{gathered}
E[<\omega, \phi>]:=\int_{S^{\prime}(\mathbb{R})}<\omega, \phi>d P(\omega)=0 \\
E\left[<\omega, \phi>^{2}\right]:=\int_{S^{\prime}(\mathbb{R})}<\omega, \phi>^{2} d P(\omega)=\|\phi\|_{L^{2}(\mathbb{R})}^{2} .
\end{gathered}
$$

Hence, we can extend the definition of $\langle\omega, \phi\rangle$ from $\phi \in S(\mathbb{R})$ to $\phi \in L^{2}(\mathbb{R})$ by using the properties (5), (6) and the facts that $S(\mathbb{R})$ is dense in $L^{2}(\mathbb{R})$ and $L^{2}(P)$ is complete. Therefore, it is natural to define

$$
\tilde{W}(t, \omega)=\tilde{W}(t)=<\omega, \chi_{[0, t]}(.)>
$$


where $\chi_{[0, t]}$ belongs to $L^{2}(\mathbb{R})$ for all $\mathrm{t}$ and defined as follows:

$$
\chi_{[0, t]}(s)= \begin{cases}1 & \text { if } s \in[0, t] \text { or } s \in[t, 0](t<0) \\ 0 & \text { otherwise }\end{cases}
$$

Then, $\tilde{W}(t)$ is a Gaussian process with mean 0 and variance t. By the Kolmogorov continuity theorem [12] it has continuous version, denoted by $W(t)=W(t, \omega)$ which is a Brownian motion. From now on we will work with the Brownian motion constructed on the white noise probability space. Note that $W(t, \omega), t \in \mathbb{R}, \omega \in \Omega=S^{\prime}(\mathbb{R})$ can be regarded as a tempered distribution and for all $\psi \in L^{2}(\mathbb{R})$,

$$
<\omega, \psi>=\int_{\mathbb{R}} \psi(t) d W(t) .
$$

We will define the filtration $\mathcal{F}_{t}$ as the $\sigma$-algebra generated by $\{W(s, \cdot), 0 \leq s \leq t\}$.

3. Review on special functions. The scope of this section is to recall some facts about special functions especially about Hermite polynomials and functions. By using them in the next section we will define an orthogonal basis for $L^{2}(P)$.

Hermite polynomials plays a crucial role in probability theory. The family of these polynomials constitute an orthogonal basis for $L^{2}(\mathbb{R}, \mu(d x))$ if the measure $\mu=\frac{1}{\sqrt{2 \pi}} e^{\frac{x^{2}}{2}}$. Hermite polynomials $h_{n}(x)$ are defined by as follows:

$$
h_{n}(x)=(-1)^{n} e^{\frac{1}{2} x^{2}} \frac{d^{n}}{d x^{n}}\left(e^{-\frac{1}{2} x^{2}}\right), x \in \mathbb{R}, n=0,1,2, \ldots
$$

Thus the first Hermite polynomials are

$$
h_{0}(x)=1, h_{1}(x)=x, h_{2}(x)=x^{2}-1, h_{3}(x)=x^{3}-3 x, \ldots
$$

Moreover,

$$
\begin{gathered}
h_{n}^{\prime}(x)=n h_{n-1}(x), n \geq 1 \\
h_{n+1}(x)=x h_{n}(x)-n h_{n-1}(x), n \geq 1
\end{gathered}
$$

are commonly used important relations between Hermite polynomials. Hermite functions, $\left\{e_{k}\right\}_{k \geq 1}$, can be described in terms of Hermite polynomials as follows:

$$
e_{k}(x)=\pi^{-1 / 4}((k-1) !)^{-\frac{1}{2}} e^{-\frac{1}{2} x^{2}} h_{k-1}(\sqrt{2} x), k=1,2,3, \ldots
$$

where $e_{k}$ is the k'th Hermite function. Moreover, note that $\left\{e_{k}\right\}_{k \geq 1}$ constitutes an orthonormal basis for $L^{2}(\mathbb{R})$ and $e_{k} \in S(\mathbb{R})$ for all $k$. They will be used in the following section for constructing the orthogonal basis for $L^{2}(P)$.

There is a practical formula proved by Itô [9] for the computation of iterated Itô integrals when the integrand is the tensor power of a given function. Then, for the tensor power of $g \in L^{2}([0, T])$ we have

$$
n ! \int_{0}^{T} \int_{0}^{t_{n}} \cdots \int_{0}^{t_{2}} g\left(t_{1}\right) g\left(t_{2}\right) \cdots g\left(t_{n}\right) d W\left(t_{1}\right) \cdots d W\left(t_{n}\right)=\|g\|^{n} h_{n}\left(\frac{\int_{0}^{T} g(t) d W(t)}{\|g\|_{L^{2}([0, T])}}\right) .
$$


Definition 1. Let $g$ be a real valued function on $L^{2}\left([0, T]^{n}\right)$. Then $n$-fold iterated Itô integrals on the interval $[0, T]$ satisfying $0 \leq t_{1} \leq t_{2} \leq \cdots \leq t_{n} \leq T$ is defined by $J_{n}$, and

$$
J_{n}(g):=\int_{0}^{T} \int_{0}^{t_{n}} \cdots \int_{0}^{t_{2}} g\left(t_{1}, t_{2}, \cdots, t_{n}\right) d W\left(t_{1}\right) \cdots d W\left(t_{n}\right) .
$$

If $g$ is symmetric function (i.e. $\left.g \in \hat{L}^{2}\left([0, T]^{n}\right)\right)$ then

$$
n ! J_{n}(g)=I_{n}(g)
$$

where indeed $I_{n}$ can be represented as follows:

$$
I_{n}(g):=\int_{0}^{T} \int_{0}^{T} \cdots \int_{0}^{T} g\left(t_{1}, t_{2}, t_{3}, \cdots, t_{n}\right) d W\left(t_{1}\right) \cdots d W\left(t_{n}\right) .
$$

4. Chaos expansions. Chaos expansion aims to represent a random variable in $L^{2}(P)$ in terms of unique fundamental functions. In this section we will present two different kinds of Wiener-Itô chaos expansion and we will state the relation between them.

\section{THEOREM 2. (Wiener-Itô chaos expansion I)}

Let $F(\omega)$ be an $F_{T}$-measurable random variable in $L^{2}(P)$, where

$$
L^{2}(P)=\left\{F: \Omega \rightarrow \mathbb{R} \text { s.t. }\|X\|_{L^{2}(P)}^{2}:=\int_{\Omega} F^{2}(\omega) d P(\omega)<\infty\right\} .
$$

Then there exists unique sequence $\left\{f_{n}\right\}_{n=0}^{\infty}$ of functions $f_{n} \in \hat{L}^{2}\left([0, T]^{n}\right)$ such that

$$
F=\sum_{n=0}^{\infty} I_{n}\left(f_{n}\right)
$$

where the convergence is in $L^{2}(P)$. Moreover, there exist the following isometry;

$$
\|F\|_{L^{2}(P)}^{2}=E\left[F^{2}\right]=\sum_{n=0}^{\infty} n !\left\|f_{n}\right\|_{L^{2}\left([0, T]^{n}\right)}^{2}
$$

Proof. The proof of this representation can be found in G. Di Nunno et al [3].

The above representation theorem is in terms of iterated Itô integrals. Let us now construct a new representation for the random variables in $L^{2}(P)$ and deal with the relations between these two representations. Define

$$
\theta_{k}(\omega):=<\omega, e_{k}>=\int_{\mathbb{R}} e_{k}(x) d W(x), \omega \in \Omega
$$

where $e_{k}$ is the k'th Hermite function defined in (9). Let $\mathcal{I}$ be the set of all finite multiindices $\alpha=\left(\alpha_{1}, \alpha_{2}, \ldots, \alpha_{m}\right)$, where $\alpha_{m} \in \mathbb{N}_{0}=\mathbb{N} \cup\{0\}, \mathrm{m}=1,2, \ldots$ and $|\alpha|=\alpha_{1}+\alpha_{2}+$ $\ldots+\alpha_{m}$. Define

$$
H_{\alpha}:=\prod_{k=1}^{m} h_{\alpha_{k}}\left(\theta_{k}(\omega)\right), \quad \omega \in \Omega
$$

The family $\left\{H_{\alpha}\right\}_{\alpha \in \mathcal{I}}$ is an orthogonal sequence that constitutes basis for the Hilbert space $L^{2}(P)$. 
THEOREM 3. (Wiener-Itô chaos expansion II)

For all $F \in L^{2}(P)$ there exists unique constants $c_{\alpha} \in \mathbb{R}$ such that

$$
F=\sum_{\alpha \in \mathcal{I}} c_{\alpha} H_{\alpha}
$$

Moreover, there exists the following isometry

$$
\|F\|_{L^{2}(P)}^{2}=\sum_{\alpha \in \mathcal{I}} \alpha ! c_{\alpha}^{2}
$$

where $\alpha !=\alpha_{1} ! \alpha_{2} ! \ldots \alpha_{m}$ ! for $\alpha=\left(\alpha_{1}, \alpha_{2}, \ldots, \alpha_{m}\right)$.

Note that by the result of Itô [9], equation (9)we obtain the following equality

$$
I_{n}\left(e^{\hat{\otimes} \alpha}\right)=\prod_{i=1}^{n} h_{\alpha_{i}}\left(\theta_{i}\right)=H_{\alpha}
$$

where $I_{n}$ is defined in (11), $\theta$ is described in the equation (13) and $H_{\alpha}$ is defined in the equation (14).

Therefore, the connection between these two expansions is if

$$
f_{n}=\sum_{\alpha \in \mathcal{I}:|\alpha|=n} c_{\alpha} e^{\hat{\otimes} \alpha}, \quad n=0,1,2, \ldots,
$$

where

and $|\alpha|=\sum_{i=1}^{m} \alpha_{i}$ then

$$
e^{\hat{\otimes} \alpha}=e_{1}^{\otimes \alpha_{1}} \hat{\otimes} e_{2}^{\otimes \alpha_{2}} \hat{\otimes} \ldots \hat{\otimes} e_{m}^{\otimes \alpha_{m}}
$$

$$
F=\sum_{n=0}^{\infty} I_{n}\left(f_{n}\right)=\sum_{\alpha \in \mathcal{I},|\alpha|=n} c_{\alpha} H_{\alpha}
$$

for some suitable constants $c_{\alpha}$. In the above equation $\otimes$ and $\hat{\otimes}$ stands for the tensor product and symmetrized tensor product, respectively. For example, if $f, g$ and $h$ are real functions on $\mathbb{R}$ then

$$
(f \otimes g \otimes h)\left(x_{1}, x_{2}, x_{3}\right)=f\left(x_{1}\right) g\left(x_{2}\right) h\left(x_{3}\right)
$$

and

$$
(f \hat{\otimes} g \hat{\otimes} h)\left(x_{1}, x_{2}, x_{3}\right)=\frac{1}{6} \sum_{\sigma} f\left(x_{\sigma_{1}}\right) g\left(x_{\sigma_{2}}\right) h\left(x_{\sigma_{3}}\right),
$$

where the sum is taken over all permutations of $\sigma$ of $1,2,3$.

5. Hida Stochastic test function and distribution space. This section contains a brief summary of (Hida) Malliavin derivative based on the representations and some useful properties of it.A useful analogy between tempered distribution space, $S^{\prime}(\mathbb{R})$ and Hida stochastic test function and distribution space on the white noise probability space will be mentioned. We will denote Hida test functions (or functionals) space and Hida distributions space with $(S)$ and $(S)^{*}$ respectively. For more information about these spaces one can see [7] and [8]. 


\section{DEFINITION 2.}

(i) $f=\sum_{\alpha \in \mathcal{I}} a_{\alpha} H_{\alpha} \in L^{2}(P)$ belongs to the Hida test function Hilbert space $(S)_{k}$ for $\mathrm{k} \in\{1,2, \ldots\}$ if

$$
\|f\|_{k}^{2}:=\sum_{\alpha \in \mathcal{I}} \alpha ! a_{\alpha}^{2}(2 \mathbb{N})^{\alpha k}<\infty
$$

where

$$
(2 \mathbb{N})^{\alpha}=\prod_{i=1}^{m}(2 i)^{\alpha_{i}}, \text { for } \alpha=\left(\alpha_{1}, \ldots, \alpha_{m}\right) \in \mathcal{I} .
$$

Then define Hida space of stochastic test functions $(S)$ as

$$
(S)=\bigcap_{k=1}^{\infty}(S)_{k},
$$

with projective topology (i.e. as $\mathrm{n}$ goes to infinity, $f_{n} \rightarrow f$ in $(S)$ iff $\left\|f_{n}-f\right\|_{k} \rightarrow 0$, for all $\left.k \in \mathbb{N}\right)$.

(ii) Similarly, let $(S)_{-q}, q=1,2, \ldots$, be the set of all expansions $F=\sum_{\alpha \in \mathcal{I}} b_{\alpha} H_{\alpha}$ such that

$$
\|F\|_{-q}^{2}:=\sum_{\alpha \in \mathcal{I}} b_{\alpha}^{2} \alpha !(2 \mathbb{N})^{-\alpha q}<\infty
$$

where $(2 \mathbb{N})^{\alpha}$ is defined in the equation (19). Then define Hida space of stochastic distributions $(S)^{*}$ as

$$
(S)^{*}=\bigcup_{q=1}^{\infty}(S)_{-q}
$$

with the inductive topology (i.e. as n goes to infinity, $F_{n} \rightarrow F$ in $(S)^{*}$ iff there exists $q$ such that $\left.\left\|F_{n}-F\right\|_{-q} \rightarrow 0\right)$.

Note that $(S)^{*}$ is the dual of $(S)$ and one can define the action of $F=\sum_{\alpha \in \mathcal{I}} b_{\alpha} H_{\alpha} \in$ $(S)^{*}$ on $f=\sum_{\alpha \in \mathcal{I}} a_{\alpha} H_{\alpha} \in L^{2}(P) \in(S)$ as follows:

$$
<F, f>=F(f)=\sum_{\alpha \in \mathcal{I}} a_{\alpha} b_{\alpha} \alpha !
$$

From the definition of these spaces we can easily extract the following inclusions.

$$
(S) \subset(S)_{k} \subset L^{2}(P) \subset(S)_{-q} \subset(S)^{*} \text {, for all } k, q .
$$

It is convenient and natural way to define Wick product on the space $(S)^{*}$.

Definition 3. If $X=\sum_{\alpha} a_{\alpha} H_{\alpha} \in(S)^{*}$ and $Y=\sum_{\beta} b_{\beta} H_{\beta} \in(S)^{*}$ then the Wick product $X \diamond Y$ of $X$ and $Y$ is defined by

$$
X \diamond Y:=\sum_{\alpha, \beta} a_{\alpha} b_{\beta} H_{\alpha+\beta}
$$

5.1. The (Hida) Malliavin Derivative. Although the Wiener space is natural space to work on when dealing with Brownian motion, this approach has some disadvantages. However, if we work on $\Omega=S^{\prime}(\mathbb{R})$ instead and follow the theory of Hida [5] then we obtain a 
complete agreement between the directional derivative and the corresponding stochastic gradient (or the Hida-Malliavin derivative). The scope of this part is to define the (Hida) Malliavin derivative and some auxiliary theorems on the white noise probability space $\left(\Omega=S^{\prime}(\mathbb{R}), \mathcal{B}, P\right)$ in order to use them in the proof of Clark-Ocone formula under change of measure. First, let us define $(S)^{*}$-integrability.

Definition 4. A function $\psi(t): \mathbb{R} \rightarrow(S)^{*}$ is $(S)^{*}$-integrable if

$$
<\psi(t), \phi>\in L^{1}(\mathbb{R})
$$

for all $\phi \in(S)$. Then the $(S)^{*}$-integral of $\psi(t)$, denoted by $\int_{\mathbb{R}} \psi(t) d t$, is the unique $(S)^{*}$ element such that

$$
<\int_{\mathbb{R}} \psi(t) d t, \phi>=\int_{\mathbb{R}}<\psi(t), \phi>d t,
$$

for all $\phi \in(S)$.

\section{DEFINITION 5.}

(i) Let $F \in(S)^{*} \supset L^{2}(P)$ be random variable and let $\gamma \in L^{2}(\mathbb{R}) \subset \Omega$ be a deterministic function. Then the directional (or Gateaux) derivative of $F$ in the direction of $\gamma$ is defined by

$$
D_{\gamma} F(\omega)=\lim _{\varepsilon \rightarrow 0} \frac{F(\omega+\varepsilon \gamma)-F(\omega)}{\varepsilon}
$$

if the limit exists in $(S)^{*}$.

(ii) Suppose there exists a function $\psi(t, \omega): \mathbb{R} \rightarrow(S)^{*}$ such that

$$
\psi(t, \omega) \gamma(t) \text { is }(S)^{*} \text {-integrable }
$$

and

$$
D_{\gamma} F(\omega)=\int_{\mathbb{R}} \psi(t, \omega) \gamma(t) d t \quad \text { for all } \gamma \in L^{2}(\mathbb{R}),
$$

then we say that $\mathrm{F}$ is (Hida)Malliavin differentiable and we put

$$
D_{t} F(\omega):=\frac{d F}{d \omega}(t, \omega)=\psi(t, \omega), \quad t \in \mathbb{R} .
$$

$D_{t} F$ is called the Hida-Malliavin derivative or the stochastic gradient of $F$ at $t$.

Example. Let $F(\omega)=<\omega, f>=\int_{\mathbb{R}} f(t) d W(t)$, for some $f \in L^{2}(\mathbb{R})$. Then

$$
\begin{aligned}
D_{\gamma} F(\omega) & =\lim _{\varepsilon \rightarrow 0} \frac{<\omega+\varepsilon \gamma, f>-<\omega, f>}{\varepsilon} \\
& =<\gamma, f>
\end{aligned}
$$

Hence, $F$ is Malliavin differentiable and $D_{t} F(\omega)=f(t)$, i.e.,

$$
D_{t}\left(\int_{\mathbb{R}} f(s) d W(s)\right)=f(t) .
$$

Definition 6. Let represent $F$ in terms of Wiener-Itô chaos expansion II, i.e. $F(\omega)=\sum_{\alpha \in \mathcal{I}} c_{\alpha} H_{\alpha} \in(S)^{*}$ then

$$
D_{t} H_{\alpha}=|\alpha| I_{|\alpha|-1}\left(e^{\hat{\otimes} \alpha}(., t)\right)
$$


or

$$
\begin{aligned}
D_{t} H_{\alpha} & =D_{t}\left(\prod_{i=1}^{m} h_{\alpha_{i}}\left(<\omega, e_{i}>\right)\right) \\
& =D_{t}\left(h_{\alpha_{1}}\left(<\omega, e_{1}>\right) h_{\alpha_{2}}\left(<\omega, e_{2}>\right) \ldots h_{\alpha_{m}}\left(<\omega, e_{m}>\right)\right) \\
& =\sum_{k=1}^{m} \prod_{i=1, j \neq k} \alpha_{k} h_{\alpha_{k}-1}\left(<\omega, e_{k}>\right) e_{k}(t) h_{\alpha_{i}}\left(<\omega, e_{i}>\right) \\
& =\sum_{k=1}^{m} \alpha_{k} e_{k}(t) H_{\alpha-\varepsilon^{k}},
\end{aligned}
$$

where $\alpha-\varepsilon^{(k)}=\left(\alpha_{1}, \alpha_{2}, \ldots, \alpha_{k}-1, \ldots, \alpha_{m}\right)$ and $e_{k}$ is the kth Hermite function.

\section{LEMMA 4.}

(i) Let $G \in(S)^{*}$. Then $D_{t} G \in(S)^{*}$ for a.a. $t \in \mathbb{R}$.

(ii) Suppose $G, G_{n} \in(S)^{*}$ for all $n \in \mathbb{N}$ and

$$
G_{n} \rightarrow G \text { in }(S)^{*} \text {. }
$$

Then there exist a subsequence $\left\{G_{n_{k}}\right\}_{k \geq 1}$ such that

$$
D_{t} G_{n_{k}} \rightarrow D_{t} G \text { in }(S)^{*},
$$

for almost all $t>0$.

Proof. (i) Let $G=\sum_{\alpha \in \mathcal{I}} c_{\alpha} H_{\alpha} \in(S)^{*}$. Then there exists a $q<\infty$ such that

$$
\begin{aligned}
\|G\|_{(S)_{-q}}^{2} & :=\sum_{\alpha \in \mathcal{I}} c_{\alpha}^{2} \alpha !(2 \mathbb{N})^{-\alpha q} \\
& =\sum_{n=0}^{\infty} \sum_{|\alpha|=n} c_{\alpha}^{2} \alpha !(2 \mathbb{N})^{-\alpha q}<\infty .
\end{aligned}
$$

By using equation (24), the Hida-Malliavin derivative of $G$ is as follows:

$$
\begin{aligned}
D_{t} G(\omega) & =\sum_{\alpha \in \mathcal{I}} c_{\alpha} D_{t}\left(H_{\alpha}(\omega)\right) \\
& =\sum_{\alpha \in \mathcal{I}} c_{\alpha} \sum_{i} \alpha_{i} H_{\alpha-\varepsilon^{(i)}}(\omega) e_{i}(t) \\
& =\sum_{\beta}\left(\sum_{i} c_{\beta+\varepsilon^{(i)}}\left(\beta_{i}+1\right) e_{i}(t)\right) H_{\beta}(\omega) \\
& :=\sum_{\beta} g_{\beta}(t) H_{\beta}(\omega),
\end{aligned}
$$

where $g_{\beta}(\omega)(t)=\sum_{i} c_{\beta+\varepsilon^{(i)}}\left(\beta_{i}+1\right) e_{i}(t)$.

We want to prove that

$$
\left\|D_{t} G\right\|_{(S)_{-q-1}}^{2}:=\sum_{n=0}^{\infty}\left(\sum_{|\beta|=n} g_{\beta}^{2} \beta !\right)(2 \mathbb{N})^{-\beta(q+1)}<\infty \text { for a.a. t. }
$$


Note that,

$$
\int_{\mathbb{R}} g_{\beta}^{2}(t) d t=\sum_{\beta} c_{\beta+\varepsilon^{(i)}}^{2}\left(\beta_{i}+1\right)^{2} .
$$

and

$$
\begin{aligned}
(2 \mathbb{N})^{-\beta} & =\prod_{i}(2 \cdot i)^{-\beta_{i}} \\
& \leq \prod_{i} e^{-\beta_{i}(\log 2)}=e^{-|\tilde{\beta}|}
\end{aligned}
$$

where $\tilde{\beta}_{i}=(\log 2) \beta_{i}$ for all $i \in \mathcal{I}$. Hence,

$$
\begin{aligned}
\int_{\mathbb{R}}\left\|D_{t} G\right\|_{(S)_{-q-1}}^{2} d t & =\sum_{\beta}\left(\sum_{i} c_{\beta+\varepsilon^{(i)}}^{2}\left(\beta_{i}+1\right)^{2}\right) \beta !(2 \mathbb{N})^{-\beta(q+1)} \\
& =\sum_{\beta}\left(\beta_{i}+1\right)(2 \mathbb{N})^{-\beta(q+1)} \sum_{\alpha,|\alpha|=|\beta|+1} c_{\alpha}^{2} \alpha ! \\
& <\sum_{n} \sum_{|\tilde{\beta}|=n}(n+1) e^{-n} \sum_{|\alpha|=(\log 2)^{-1} n+1}^{2} \alpha !(2 \mathbb{N})^{-\alpha q}
\end{aligned}
$$

Using the fact that $(n+1) e^{-n} \leq 1$ for all $n$, we get

$$
\begin{aligned}
\int_{\mathbb{R}}\left\|D_{t} G\right\|_{(S)_{-q-1}}^{2} d t & <\sum_{n}\left(\sum_{|\alpha|=(\log 2)^{-1} n+1} c_{\alpha}^{2} \alpha !\right)(2 \mathbb{N})^{-\alpha q} \\
& \leq\|G\|_{(S)_{-q}}<\infty
\end{aligned}
$$

Therefore, $D_{t} G \in(S)_{-q-1} \subset(S)^{*}$ for a.a. $t$.

(ii) To prove this part, it sufficies to prove that if $G_{n} \rightarrow 0$ in $(S)_{-q}$, then there exist a subsequence $\left\{G_{n_{k}}\right\}_{k \geq 1}$ such that $D_{t} G_{n_{k}} \rightarrow 0$ in $(S)^{*}$ as $k$ goes to infty, for a.a. t. We have proved that

$$
\int_{\mathbb{R}}\left\|D_{t} G_{n}\right\|_{(S)_{-q-1}}^{2} d t \leq\left\|G_{n}\right\|_{(S)_{-q}}^{2} \rightarrow 0
$$

Therefore,

$$
\left\|D_{t} G_{n}\right\|_{(S)_{-q-1}} \rightarrow 0 \quad \text { in } \quad L^{2}(\mathbb{R}) .
$$

So, there exists a subsequence $\left\{\left\|D_{t} G_{n_{k}}\right\|_{(S)_{-q-1}}\right\}_{k \geq 1}$ such that $\left\|D_{t} G_{n_{k}}\right\|_{(S)_{-q-1}} \rightarrow 0$ for a.a. $t$ as $k \rightarrow \infty$.

Let

$$
F=P(x)=\sum_{\alpha} c_{\alpha} x^{\alpha}
$$

be a polynomial and the Wick product version of $F$ can be written as

$$
F=P^{\diamond}(X)=\sum_{\alpha} X^{\diamond \alpha}
$$

where $\alpha=\left(\alpha_{1}, \alpha_{2}, \ldots, \alpha_{m}\right) \in \mathcal{I}, X^{\diamond \alpha}(\omega)=\left(X_{1}^{\diamond \alpha_{1}} \diamond \ldots \diamond X_{m}^{\diamond \alpha_{m}}\right)$ where $X_{i}(\omega)=<\omega, e_{i}>$. Note that under this settlement, $X^{\diamond \alpha}(\omega)=H_{\alpha}(\omega)$ for all multi indices $\alpha$.

THEOREM 5. (Chain rule for polynomials)

Suppose $P(x)=\sum_{\alpha} c_{\alpha} x^{\alpha}$ be a $n$ variables polynomial. Then $P(X)$ is (Hida)Malliavin 
differentiable and

$$
\begin{aligned}
D_{t} P(X) & =\sum_{i=1}^{n} \frac{\partial P}{\partial X_{i}}\left(X_{1}, \ldots, X_{n}\right) e_{i}(t) \\
& =\sum_{i=1}^{n} \frac{\partial P}{\partial X_{i}}\left(X_{1}, \ldots, X_{n}\right) D_{t}\left(X_{i}\right) .
\end{aligned}
$$

Proof. In this proof we will use the definition of directional derivative of $F$ in the direction $\gamma \in L^{2}(\mathbb{R})$. Put $F(\omega)=P(X(\omega))$.

$$
\begin{aligned}
D_{\gamma} P(X) & =D_{\gamma} F \\
& =\lim _{\varepsilon \rightarrow 0} \frac{F(\omega+\varepsilon \gamma)-F(\omega)}{\varepsilon} \\
& =\lim _{\varepsilon \rightarrow 0} \frac{P(X(\omega+\varepsilon \gamma))-P(X(\omega))}{\varepsilon} \\
& =\lim _{\varepsilon \rightarrow 0} \frac{P\left(X(\omega)+\varepsilon<\gamma, e_{i}>\right)-P(X(\omega))}{\varepsilon} \\
& =\sum_{i=1}^{n} \frac{\partial P}{\partial x_{i}}(X(\omega))<\gamma, e_{i}>
\end{aligned}
$$

So we can conclude that $D_{\gamma} F(\omega)=\int_{\mathbb{R}}\left(\sum_{i=1}^{n} \frac{\partial P}{\partial x_{i}}(X(\omega)) e_{i}(t)\right) \gamma(t) d t$. This proves that $F=P(X)$ is also (Hida)-Malliavin differentiable and (28) holds.

THEOREM 6. (Chain rule)

Suppose $F \in(S)^{*} \supset L^{2}(\lambda \times P)$ is Malliavin differentiable. Let $\Phi \in \mathcal{C}^{1}(\mathbb{R})$. Then $\Phi(F)$ is Malliavin differentiable and

$$
D_{t}(\Phi(F))=\Phi^{\prime}(F) D_{t} F .
$$

Proof. This can be easily proved by using Lemma 4 . This representation is the extension of Chain rule for polynomials.

More generally, if $F_{1}, F_{2}, \ldots, F_{m}$ are Malliavin differentiable then

$$
D_{t}\left(\Phi\left(F_{1}, F_{2}, \ldots, F_{m}\right)\right)=\sum_{i=1}^{m} \frac{\partial \Phi}{\partial F_{i}}\left(F_{1}, F_{2}, \ldots, F_{m}\right) \cdot D_{t} F_{i}
$$

\section{REMARK 1.}

(i) Note that as a consequence of general chain rule we can easily write product rule, i.e.,

$$
D_{t}\left(F_{1} F_{2}\right)=F_{1} D_{t} F_{2}+F_{2} D_{t} F_{1}
$$

(ii) Another result of chain rule is

$$
\begin{aligned}
D_{t}\left(h_{n}(<\omega, f>)\right) & =h_{n}^{\prime}(<\omega, f>) f(t) \\
& =n h_{n-1}(<\omega, f>) f(t),
\end{aligned}
$$

where $\left\{h_{n}(x)\right\}_{n \geq 0}$ are the Hermite polynomials. 
LEMma 7. Let $F=I_{n}\left(f_{n}\right) \in L^{2}(P)$ for some $f_{n} \in \hat{L}^{2}\left([0, T]^{n}\right)$. Then

$$
D_{t} F=n I_{n-1}\left(f_{n}(., t)\right) \text {, }
$$

where $I_{n-1}\left(f_{n}(., t)\right)$ stands for the $(n-1)$-iterated Itô integral which is defined with respect to the $n-1$ first variables $t_{1}, t_{2}, \ldots, t_{n-1}$ of $f_{n}\left(t_{1}, t_{2}, \ldots, t_{n-1}, t\right)$.

Proof. Here we will give the sketch of the proof. Let us firstly assume $f_{n}=f^{\otimes n}$ for some $f \in L^{2}([0, T])$. Then by equation (10),

Hence by Remark (ii)

$$
I_{n}\left(f_{n}\right)=\|f\|^{n} h_{n}\left(\frac{<\omega, f>}{\|f\|}\right) .
$$

$$
\begin{aligned}
D_{t} I_{n}\left(f_{n}\right) & =D_{t}\left(\|f\|^{n} h_{n}\left(\frac{<\omega, f>}{\|f\|}\right)\right) \\
& =n\|f\|^{n} h_{n-1}\left(\frac{<\omega, f>}{\|f\|}\right) \frac{f(t)}{\|f\|} \\
& =n\|f\|^{n-1} h_{n-1}\left(\frac{<\omega, f>}{\|f\|}\right) f(t) \\
& =n I_{n-1}\left(f_{n}(., t)\right)
\end{aligned}
$$

Secondly, suppose $f_{n}$ has the form

$$
f_{n}=e_{1}^{\hat{\otimes} \alpha_{1}} \hat{\otimes} e_{2}^{\hat{\otimes} \alpha_{2}} \hat{\otimes} \ldots \hat{\otimes} e_{k}^{\hat{\otimes} \alpha_{k}}
$$

where $|\alpha|=\alpha_{1}+\alpha_{2}+\ldots+\alpha_{k}=n$ for $\alpha=\left(\alpha_{1}, \ldots, \alpha_{k}\right), \hat{\otimes}$ denotes the symmetrized tensor product and $\left\{e_{k}\right\}_{k \geq 1}$ is the family of hermite functions which are orthonormal basis of $L^{2}(\mathbb{R})$. By equation (10),

$$
I_{n}\left(f_{n}\right)=h_{\alpha_{1}}\left(<\omega, e_{1}>\right) \cdots h_{\alpha_{k}}\left(<\omega, e_{k}>\right)
$$

then the equality (31) holds again by using chain rule. Since any function $f_{n} \in \hat{L}^{2}\left([0, T]^{n}\right)$ can be approximated in $L^{2}\left([0, T]^{n}\right)$ by linear combinations of basis of the form given by (33) the proof is completed.

Hence, Hida-Malliavin derivative defined above coincides with the Malliavin derivative on the stochastic Sobolev space, $\mathbb{D}_{1,2}$ with Wiener space settlement for this case.

6. The Clark-Ocone formula under change of measure. In this section, we will prove the extended Clark-Ocone formula under change of measure by using the white noise theory. We will work on the space $\mathcal{G}^{*} \supset L^{2}(P)$, the space of stochastic distributions. The advantage of using $\mathcal{G}^{*}$ lies in the fact that it is convenient space to work with the conditional expectation. Moreover, $D_{t} F$ is not well defined in the classical sense, because we have not assumed that $F \in \mathbb{D}_{1,2}$. Therefore, $D_{t} F$ is assumed to be an element of $(S)^{*}$, where $E\left[D_{t} F \mid \mathcal{F}_{t}\right] \in L^{2}([0, T] \times P)$.

6.1.The construction of the spaces $\mathcal{G}$ and $\mathcal{G}^{*}$ In this subsection we will follow the theory in T. Hida et al. [7] and K. Aase et al. [1]. 


\section{Definition 7.}

(i) Let $\lambda \in \mathbb{R}$. The space $\mathcal{G}_{\lambda}$ consists of all formal expansions

$$
F=\sum_{n=0}^{\infty} I_{n}\left(f_{n}\right)
$$

such that

$$
\|F\|_{\mathcal{G}_{\lambda}}^{2}:=\sum_{n=0}^{\infty} n !\left\|f_{n}\right\|_{L^{2}\left(\mathbb{R}^{n}\right)}^{2} e^{2 \lambda n}<\infty .
$$

(ii) Define

$$
\mathcal{G}=\bigcap_{\lambda \in \mathbb{R}} \mathcal{G}_{\lambda}
$$

equip with the projective topology and

$$
\mathcal{G}^{*}=\bigcup_{\lambda \in \mathbb{R}} \mathcal{G}_{\lambda}
$$

equip with inductive topology. Then $\mathcal{G}^{*}$ is the dual of $\mathcal{G}$, and the action of $Y=\sum_{n \geq \infty} I_{n}\left(f_{n}\right) \in \mathcal{G}^{*}$ on $X=\sum_{m \geq \infty} I_{m}\left(g_{m}\right) \in \mathcal{G}$ is

$$
<Y, X>_{\mathcal{G}, \mathcal{G}^{*}}=\sum_{n=0}^{\infty} n !\left(f_{n}, g_{n}\right)_{L^{2}\left(\mathbb{R}^{n}\right)} .
$$

Lemma 8 .

(i) Suppose $F \in \mathcal{G}^{*}$. Then $D_{t} F \in \mathcal{G}^{*}$ for a.a. $t \in \mathbb{R}$.

(ii) Suppose $F, F_{n} \in \mathcal{G}^{*}$ for all $n \in \mathbb{N}$ and

$$
F_{n} \rightarrow F \text { in } \mathcal{G}^{*} \text {. }
$$

Then there exists a subsequence $\left\{F_{n_{k}}\right\}_{k \geq 1}$ such that

$$
D_{t} F_{n_{k}} \rightarrow D_{t} F \text { in } \mathcal{G}^{*}
$$

for almost all $t>0$.

Proof. The proof and further details can be found in K. Aase et al [1].

The following theorem plays a crucial role for proving the Clark-Ocone formula under change of measure.

Theorem 9. Let $F=\sum_{n=0}^{\infty} I_{n}\left(f_{n}\right) \in L^{2}(P)$. Then $D_{t} F \in \mathcal{G}^{*}$ and it is defined as

$$
D_{t} F=\sum_{n=1}^{\infty} n I_{n-1}\left(f_{n}(., t)\right) \text {. }
$$

Proof. Let $F \in L^{2}(P)$. Define $F_{m}=\sum_{n=0}^{m} I_{n}\left(f_{n}\right)$. Then $F_{m} \rightarrow F$ in $L^{2}(P)$ which implies $F_{m} \rightarrow F$ in $\mathcal{G}^{*}$. By Lemma 6.1., there exists a subsequence $F_{m_{k}}$ such that $D_{t} F_{m_{k}} \rightarrow D_{t} F$ in $\mathcal{G}^{*}$, i.e.,

$$
\sum_{n=0}^{m_{k}} n I_{n-1}\left(f_{n}(., t)\right) \rightarrow D_{t} F
$$


in $\mathcal{G}^{*}$. We want to prove that

$$
\sum_{n=0}^{m_{k}} n I_{n-1}\left(f_{n}(., t)\right) \rightarrow \sum_{n=0}^{\infty} n I_{n-1}\left(f_{n}(., t)\right)
$$

in $\mathcal{G}^{*}$ as $m_{k}$ goes to infinity. Consider

$$
\left\|\sum_{m_{k+1}}^{\infty} n I_{n-1}\left(f_{n}(., t)\right)\right\|_{\mathcal{G}_{-q-1}}^{2}=\sum_{m_{k+1}}^{\infty}(n-1) ! n^{2}\left\|f_{n}(., t)\right\|_{L^{2}\left(\mathbb{R}^{n-1}\right)}^{2} e^{-2 q(n-1)} .
$$

Then taking the integral of both sides,

$$
\begin{aligned}
\int_{\mathbb{R}}\left\|\sum_{m_{k+1}}^{\infty} n I_{n-1}\left(f_{n}(., t)\right)\right\|_{\mathcal{G}_{-q}}^{2} d t & =\sum_{m_{k+1}}^{\infty} n n !\left\|f_{n}(., t)\right\|_{L^{2}\left(\mathbb{R}^{n}\right)}^{2} e^{-2 q(n-1)} \\
& \leq K \sum_{m_{k+1}}^{\infty} n !\left\|f_{n}\right\|_{L^{2}\left(\mathbb{R}^{n}\right)}^{2} \\
& =K\|F\|_{L^{2}(P)}<\infty
\end{aligned}
$$

where $\mathrm{K}$ is a constant.

\subsection{Conditional Expectation in $\mathcal{G}^{*}$}

Definition 8. Let $F=\sum_{n=0}^{\infty} I_{n}\left(f_{n}\right) \in \mathcal{G}^{*}$. Then the conditional expectation of $F$ with respect to filtration $\mathcal{F}_{t}$ is defined by

$$
E\left[F \mid F_{t}\right]=\sum_{n=0}^{\infty} I_{n}\left(f_{n} \chi_{[0, t] \otimes n}\right) .
$$

Note that this coincides with the usual expectation if $F \in L^{2}(P)$. Moreover, since

$$
\left\|E\left[F \mid \mathcal{F}_{t}\right]\right\|_{\mathcal{G}_{\lambda}} \leq\|G\|_{\mathcal{G}_{\lambda}} \text {, for all } \lambda \in \mathbb{R}
$$

then

$$
E\left[F \mid \mathcal{F}_{t}\right] \in \mathcal{G}^{*}
$$

Proposition 10. If $F \in \mathcal{G}^{*}$ then $E\left[F \mid \mathcal{F}_{t}\right] \in \mathcal{G}^{*}$ and

$$
D_{s} E\left[F \mid \mathcal{F}_{t}\right]=E\left[D_{s} F \mid \mathcal{F}_{t}\right] \chi_{[0, t]}(s) .
$$

Proof. We begin by proving this equality for $F=I_{n}\left(f_{n}\right) \in \mathcal{G}^{*}$. By using equation (39) and Lemma 7, we have

$$
\begin{aligned}
D_{s} E\left[F \mid \mathcal{F}_{t}\right] & =D_{s} E\left[I_{n}\left(f_{n}\right) \mid \mathcal{F}_{t}\right] \\
& =D_{s}\left(I_{n}\left(f_{n} \chi_{[0, t]}^{\otimes n}\right)\right) \\
& =n I_{n-1}\left(f_{n}(., s) \chi_{[0, t]}^{\otimes(n-1)}(.)\right) \chi_{[0, t]}(s) \\
& =E\left[D_{s} F \mid \mathcal{F}_{t}\right] \chi_{[0, t]}(s)
\end{aligned}
$$


Next, assume $F=\sum_{n=0}^{\infty} I_{n}\left(f_{n}\right) \in \mathcal{G}^{*}$. Then by definition, $E\left[F \mid \mathcal{F}_{t}\right]=\sum_{n=0}^{\infty} I_{n}\left(f_{n} \chi_{[0, t]}^{\otimes n}\right)$. Consider,

$$
\begin{aligned}
\left\|E\left[F \mid \mathcal{F}_{t}\right]\right\|_{\mathcal{G}_{-q}}^{2} & =\sum_{n=0}^{\infty} n !\left\|f_{n} \chi_{[0, t]}^{\otimes n}\right\|_{L^{2}\left(\mathbb{R}^{n}\right)}^{2} e^{-2 q n} \\
& <\sum_{n=0}^{\infty} n !\left\|f_{n}\right\|_{L^{2}\left(\mathbb{R}^{n}\right)}^{2} \\
& =\|F\|_{\mathcal{G}_{-q}}^{2}<\infty
\end{aligned}
$$

Therefore, if $E\left[F \mid \mathcal{F}_{t}\right] \in \mathcal{G}^{*}$ and

$$
\begin{aligned}
D_{s}\left(E\left[F \mid \mathcal{F}_{t}\right]\right) & =\sum_{n=1}^{\infty} n I_{n-1}\left(f_{n}(., s) \chi_{[0, t]}^{\otimes(n-1)}\right) \chi_{[0, t]}(s) \\
& =E\left[D_{s} F \mid \mathcal{F}_{t}\right] \chi_{[0, t]}(s) .
\end{aligned}
$$

Corollary 11. Let $u=u(t) \in L^{2}(P), t \in[0, T]$, be an $\mathbb{F}$-adapted stochastic process. Then

(i) $D_{s} u(t)$ is $\mathbb{F}$ adapted for all $s$,

(ii) $D_{s} u(t)=0$ for $s>t$.

THEOREM 12. (Fundamental theorem of stochastic calculus)

Let $u \in L^{2}(P)$ be a stochastic process satisfying the following conditions:

(i) $E\left[\int_{0}^{T} u(s)^{2} d s\right]<\infty$

(ii) $s \rightarrow D_{t} u(s)$ is $(S)^{*}$-integrable, for all $t \in[0, T]$

(iii) $E\left[\int_{0}^{T}\left(\int_{0}^{T} D_{t}(u(s)) \delta W(s)\right)^{2} d t\right]<\infty$

then $\delta(u)=\int_{0}^{T} u(s) \delta W(s) \in L^{2}(P)$ and

$$
D_{t}\left(\int_{0}^{T} u(s) \delta W(s)\right)=\int_{0}^{T} D_{t} u(s) \delta W(s)+u(t) .
$$

Proof. Let us firstly assume that $u(s)=I_{n}\left(f_{n}(., s)\right)$, where $f_{n}(., s) \in L^{2}\left([0, T]^{n+1}\right)$ and $f_{n}(., s)$ is symmetric with respect to the first $n$ variables. Then by definition, the skorohod integral of $u(s)$ is

$$
\delta(u)=I_{n+1}\left(\tilde{f}_{n}\left(t_{1}, t_{2}, \cdots, t_{n+1}\right)\right),
$$

where

$$
\tilde{f}_{n}\left(t_{1}, t_{2}, \cdots, t_{n+1}\right)=\frac{1}{n+1}\left[f_{n}\left(\cdot, t_{1}\right)+\ldots+f_{n}\left(\cdot, t_{n+1}\right)\right] .
$$

Then,

$$
\begin{aligned}
D_{t} \delta(u) & =(n+1) I_{n}\left(\tilde{f}_{n}(\cdot, t)\right) \\
& =(n+1) I_{n}\left(\frac{1}{n+1}\left[f_{n}\left(t, \cdot, t_{1}\right)+\ldots+f_{n}\left(t, \cdot, t_{n}\right)+f_{n}(\cdot, \cdot, t)\right]\right) \\
& =I_{n}\left(f_{n}\left(t, \cdot, t_{1}\right)+\ldots+f_{n}\left(t, \cdot, t_{n}\right)+f_{n}(\cdot, \cdot, t)\right) .
\end{aligned}
$$


On the other hand,

$$
\begin{aligned}
\delta\left(D_{t} u\right) & =\int_{0}^{T} D_{t} u(s) \delta W(s) \\
& =\int_{0}^{T} n I_{n-1}\left(f_{n}(\cdot, t, s)\right) \delta W(s) \\
& =n I_{n}\left(\hat{f}_{n}(\cdot, t, \cdot)\right)
\end{aligned}
$$

where

$$
\hat{f}_{n}\left(t_{1}, \cdots, t_{n-1}, t, t_{n}\right)=\frac{1}{n}\left[f_{n}\left(t, \cdot, t_{1}\right)+\cdots+f_{n}\left(t, \cdot, t_{n}\right)\right]
$$

Therefore,

$$
\delta\left(D_{t} u\right)=I_{n}\left(f_{n}\left(t, \cdot t_{1}\right)+\cdots+f_{n}\left(t, \cdot t_{n}\right)\right) .
$$

Hence, $D_{t}(\delta(u))-\delta\left(D_{t}(u)\right)=u(t)$, which completes the first part of the proof. Next, assume $u(s)$ is the infinite summation of iterated integrals, i.e., $u(s)=\sum_{n=0}^{\infty} I_{n}(f)$. Define $u_{m}(s)=\sum_{n=0}^{m} I_{n}\left(f_{n}(\cdot, s)\right)$. Then $u_{m} \rightarrow u$ in $L^{2}(P)$ which implies $u_{m} \rightarrow u$ in $\mathcal{G}^{*}$. By Lemma 8, there exists a subsequence $\left\{u_{m_{k}}\right\}_{k \geq 1}$ such that $D_{t}\left(u_{m_{k}}\right) \rightarrow D_{t}(u)$ in $\mathcal{G}^{*}$. Since Skorohod integral of random variable in $\mathcal{G}^{*}$ is in $(S)^{*}$, (i.e. $\left.\delta\left(D_{t}\left(u_{m_{k}}\right)\right) \in(S)^{*}\right)$ by assumption (ii) and lemma for Skorohod integrals then

$$
\int_{0}^{T}\left\|D_{t}(\delta(u))-D_{t}\left(\delta\left(u_{m_{k}}\right)\right)\right\|_{(S)_{-q}}^{2} d t \rightarrow 0
$$

and by Lemma 4,

$$
\int_{0}^{T}\left\|\delta\left(D_{t} u\right)-\delta\left(D_{t} u_{m_{k}}\right)\right\|_{(S)_{-q}}^{2} d t \rightarrow 0 .
$$

COROllary 13. Let $u$ be as in previous theorem and in addition to this assume $u(s)$ is $\mathbb{F}$-adapted then

$$
D_{t}\left(\int_{0}^{T} u(s) d W(s)\right)=\int_{t}^{T} D_{t} u(s) d W(s)+u(t)
$$

\subsection{The Clark-Ocone theorem for $L^{2}(P)$}

Theorem 14. Let $\lambda$ denote the Lebesgue measure on $\mathbb{R}$. Suppose $F(\omega) \in L^{2}(P)$ be $\mathcal{F}_{T^{-}}$ measurable. Then

$$
(t, \omega) \rightarrow E\left[D_{t} F \mid \mathcal{F}_{t}\right](\omega) \in L^{2}(\lambda \times P)
$$

and

$$
F(\omega)=E[F]+\int_{0}^{T} E\left[D_{t} F \mid \mathcal{F}_{t}\right] d W(t)
$$

Proof. The proof can be found in K. Aase et al [1].

Now our aim is to derive the formula when the probability measure is changed.

6.4.The Clark-Ocone theorem under change of measure for $L^{2}(P)$ 


\section{THEOREM 15. (Girsanov)}

Let $W(t)$ be a Brownian motion on the white noise probability space $(\Omega, \mathcal{F}, P)$. Let $(u(t))_{0 \leq t \leq}$ be an adapted measurable process satisfying $\int_{0}^{T} u^{2}(s) d s<\infty$ a.s. and such that $Z(T)$ defined by

$$
Z(T)=\exp \left\{-\int_{0}^{T} u(s) d W(s)-\frac{1}{2} \int_{0}^{T} u^{2}(s) d s\right\}
$$

is a martingale. Then under the white noise probability measure $Q$ with density $Z(T)$ relative to $P$, the process $\hat{W}(t)$ defined by $\hat{W}(t)=W(t)+\int_{0}^{t} u(s) d s$ is a Brownian motion under measure $Q$.

Lemma 16. Let $F \in L^{2}(P)$ be $\mathcal{F}_{T}$-measurable, $Q$ and $Z(T)$ is defined as in Girsanov theorem. Then

$$
D_{t}(Z(T) F)=Z(T)\left\{D_{t} F-F\left[u(t)+\int_{t}^{T} D_{t} u(s) d \hat{W}(s)\right]\right\}
$$

Proof. By the product rule,

$$
D_{t}(Z(T) F)=D_{t} F Z(T)+D_{t} Z(t) F .
$$

So it remains to find the (Hida)Malliavin derivative of $Z(T)$. By the Theorem 6 , Corollary 11 and Corollary 13,

$$
\begin{aligned}
D_{t} Z(T) & =Z(T)\left\{-D_{t}\left(\int_{0}^{T} u(s) d W(s)\right)-\frac{1}{2} D_{t}\left(\int_{0}^{T} u^{2}(s)\right)\right\} \\
& =Z(T)\left\{-\left(\int_{t}^{T} D_{t} u(s) d W(s)+u(t)\right)-\int_{0}^{T} u(s) D_{t} u(s) d s\right\} \\
& =Z(T)\left\{-\int_{t}^{T} D_{t} u(s) d \hat{W}(s)-u(t)\right\}
\end{aligned}
$$

After setting the necessary theory on white noise analysis we are ready to define and prove the Clark-Ocone formula under change of measure formula.

THEOREM 17. (The Clark-Ocone formula under change of measure for $L^{2}(P)$ ) Suppose $F \in L^{2}(P)$ is $\mathcal{F}_{T}$-measurable and that

$$
\begin{aligned}
E_{Q}[|F|] & <\infty \\
E_{Q}\left[\int_{0}^{T}\left|D_{t} F\right|^{2} d t\right] & <\infty \\
E_{Q}\left[|F| \int_{0}^{T}\left(\int_{0}^{T} D_{t} u(s) d W(s)+\int_{0}^{T} u(s) D_{t} u(s) d s\right)^{2} d t\right] & <\infty
\end{aligned}
$$

Then

$$
F(\omega)=E_{Q}[F]+\int_{0}^{T} E_{Q}\left[\left(D_{t} F-F \int_{t}^{T} D_{t} u(s) d \hat{W}(s)\right) \mid \mathcal{F}_{t}\right] d \hat{W}(t),
$$

where $\hat{W}(t)$ is a Brownian motion under white noise probability measure $Q$ and $D_{t} F \in \mathcal{G}^{*}$ is Hida-Malliavin derivative. 
Proof. Let $Y(t)=E_{Q}\left[F \mid \mathcal{F}_{t}\right]$. Note that

$$
E_{Q}\left[F \mid \mathcal{F}_{0}\right]=E_{Q}[F]
$$

and

$$
E_{Q}\left[F \mid \mathcal{F}_{T}\right]=F
$$

Let us define

$$
\lambda(t)=Z^{-1}(t)=\exp \left\{\int_{0}^{t} u(s) d W(s)+\frac{1}{2} \int_{0}^{t} u^{2}(s) d s\right\} .
$$

Then by Itô Formula,

$$
d \lambda(t)=\lambda(t) u(s) d \hat{W}(s) .
$$

Using Bayes' formula (Karatzas and Shreve Lemma 3.5.3. [11]) and the settlement of $Y(t)$,

$$
\begin{aligned}
Y(t) & =E\left[Z(T) F \mid \mathcal{F}_{t}\right] Z^{-1}(t) \\
& =\lambda(t) E\left[Z(T) F \mid \mathcal{F}_{t}\right] .
\end{aligned}
$$

If we apply Clark-Ocone formula for the random variable $E\left[Z(T) F \mid \mathcal{F}_{t}\right] \in L^{2}(P)$, we get

$$
\begin{aligned}
E\left[Z(T) F \mid \mathcal{F}_{t}\right] & =E[Z(T) F]+\int_{0}^{t} E\left[D_{s}(Z(T) F) \mid \mathcal{F}_{s}\right] d W(s) \\
& :=U(t) .
\end{aligned}
$$

Hence, $Y(t)=\lambda(t) U(t)$ and by using Bayes' formula, Lemma 16 we have the following:

$$
\begin{aligned}
d Y(t)= & \lambda(t) d U(t)+U(t) d \lambda(t)+d<\lambda, U>_{t} \\
= & \left\{E_{Q}\left[D_{t} F \mid \mathcal{F}_{t}\right]-E_{Q}\left[F u(t) \mid \mathcal{F}_{t}\right]\right. \\
& \left.-E_{Q}\left[F \int_{t}^{T} D_{t} u(s) d \hat{W}(s) \mid \mathcal{F}_{t}\right]+u(t) E_{Q}\left[F \mid \mathcal{F}_{t}\right]\right\} d \hat{W}(t) .
\end{aligned}
$$

If we integrate both sides on $[0, T]$, the proof will be completed.

7. Application to finance. In this section we will demonstrate how extended ClarkOcone theorem under change of measure can be applied in portfolio optimization. The main advantage of this setting is that $F$ need not be in $\mathbb{D}_{1,2}$. Let us assume that we have two possible investments which are a risk free asset, bond, and a risky asset, stock. Moreover, suppose the prices of these two financial instrument follow the following stochastic differential equations under probability measure P;

(i) Risk free asset (Bond)

$$
\begin{aligned}
d S_{0}(t) & =\rho(t) S_{0}(t) d t \\
S_{0}(0) & =1 .
\end{aligned}
$$


(ii) Risky asset (Stock)

$$
\begin{aligned}
d S_{1}(t) & =\mu(t) S_{1}(t) d t+\sigma(t) S_{1}(t) d W(t) \\
S_{1}(0) & >0 .
\end{aligned}
$$

Here $\rho(t)=\rho(t, \omega), \mu(t)=\mu(t, \omega)$ and $\sigma(t)=\sigma(t, \omega), \omega \in \Omega$ are $\mathcal{F}_{t}$-measurable processes for all $t \geq 0$ satisfying the following condition,

$$
E\left[\int_{0}^{T}\left\{|\rho(t)|+|\mu(t)|+\sigma^{2}(t)\right\} d t\right]<\infty
$$

Moreover, suppose in the economy there exists a contingent-claim. In this paper, we will deal with a digital option which has a payoff function at the maturity

$$
F=\chi_{[K, \infty)}(W(T)),
$$

where $K$ is the exercise price of this contingent-claim. Our aim is to find the replicating portfolio for this option where at the maturity the value of the portfolio is equal to the payoff function. If $\theta(t)=\theta(t, \omega)=\left(\theta_{0}(t), \theta_{1}(t)\right), \omega \in \Omega$, denotes the number of the unites invested at time $\mathrm{t}$ in risk free and risky assets respectively, then the value of the portfolio will be

$$
V^{\theta}(t)=\theta_{0}(t) S_{0}(t)+\theta_{1}(t) S_{1}(t)
$$

For computational purposes it is often convenient to assume the portfolio self-financing, i.e.,

$$
d V^{\theta}=\theta_{0}(t) d S_{0}(t)+\theta_{1}(t) d S_{1}(t) .
$$

Then the value of the portfolio at time $\mathrm{t}$ can be represented as follows:

$$
d V^{\theta}(t)=\left[\rho(t) V^{\theta}(t)+(\mu(t)-\rho(t)) \theta_{1}(t) S_{1}(t)\right] d t+\sigma(t) \theta_{1}(t) S_{1}(t) d W(t) .
$$

There are various ways to define market price of risk. It is convenient to define market price of risk, $u(t)$ as follows

$$
\mu(t)-\rho(t)=\sigma(t) u(t)
$$

or equivalently

$$
u(t)=\frac{\mu(t)-\rho(t)}{\sigma(t)} .
$$

Then by Girsanov theorem,

$$
\hat{W}(t)=W(t)+\int_{0}^{t} u(t) d t
$$

is a Wiener process with respect to the measure $Q$. Then using equation (53) we can rewrite the equation (52)in terms of the Wiener process $\hat{W}(t)$,

$$
d V^{\theta}(t)=\rho(t) V^{\theta}(t) d t+\sigma(t) \theta_{1}(t) S_{1}(t) d \hat{W}(t) .
$$

Define discounted value of the portfolio $U^{\pi}(t), \forall t \in[0, T]$,

$$
U^{\theta}(T)=e^{-\int_{0}^{T} \rho(s) d s} V^{\pi}(T)=V^{\theta}(0)+\int_{0}^{T} e^{-\int_{0}^{t} \rho(s) d s} \sigma(t) \theta_{1}(t) S_{1}(t) d \hat{W}(t) .
$$


If we apply Clark-Ocone formula under change of measure for $U^{\theta}(t), \forall t \in[0, T]$,

$$
U^{\theta}(T)=E_{Q}\left[U^{\theta}(T)\right]+\int_{0}^{T} E_{Q}\left[\left(D_{t} U^{\pi}(T)-U^{\theta}(T) \int_{t}^{T} D_{t} u(s) d \hat{W}(s)\right) \mid \mathcal{F}_{t}\right] d \hat{W}(t) .
$$

Comparing the terms in equations (54) and (55), the number of risky assets in the replicating portfolio can be found as follows:

$\theta_{1}(t)=e^{\int_{0}^{t} \rho(s) d s} \sigma^{-1}(t) S_{1}^{-1} E_{Q}\left[\left(D_{t}\left(e^{-\int_{0}^{T} \rho(s) d s} F\right)-e^{-\int_{0}^{T} \rho(s) d s} F \int_{t}^{T} D_{t} u(s) d \hat{W}(s)\right) \mid \mathcal{F}_{t}\right]$.

In particular, if we choose $\rho$ constant and $\mu, \sigma$ deterministic functions then the equation turns out to be

$$
\theta_{1}(t)=e^{-\rho(T-t)} \sigma^{-1}(t) S_{1}^{-1} E_{Q}\left[D_{t} F \mid \mathcal{F}_{t}\right]
$$

where $F=\chi_{[K, \infty)}(W(T))$. In order to calculate $E_{Q}\left[D_{t}\left(\chi_{[K, \infty)}(W(T))\right) \mid \mathcal{F}_{t}\right]$ we will use the Donsker delta function. For more information we refer to K. Aase et al. [2].

Definition 9. Let $Y: \Omega \rightarrow \mathbb{R}$ be a random variable which belongs to $\mathcal{G}^{*}$. Then the continuous function

$$
\delta_{Y}(.): \mathbb{R} \rightarrow \mathcal{G}^{*}
$$

is called Donsker delta function of $\mathrm{Y}$ if it has the following property,

$$
\int_{\mathbb{R}} g(y) \delta_{Y}(y) d y=g(Y) \text { a.s. }
$$

for all measurable function $g: \mathbb{R} \rightarrow \mathbb{R}$ such that the integral converges.

Theorem 18. Let $\phi:[0, T] \rightarrow \mathbb{R}$ and $\alpha:[0, T] \rightarrow \mathbb{R}$ be deterministic functions such that $\|\phi\|_{L^{2}([0, T])}$ and $\|\alpha\|_{L^{2}([0, T])}<\infty$. Define

$$
Y(t)=Y(t, \omega)=\int_{0}^{t} \phi(s) d \hat{W}(s)+\int_{0}^{t} \phi(s) \alpha(s) d s .
$$

Let $g: \mathbb{R} \rightarrow \mathbb{R}$ be bounded. Then

$$
f(Y(T))=V_{0}+\int_{0}^{T} \Psi(t, \omega) \diamond\left(\alpha(t)+\dot{\hat{W}}_{t}\right) d t
$$

where

$$
\begin{gathered}
V_{0}=\int_{\mathbb{R}} \frac{g(y)}{\sqrt{2 \pi}\|\phi\|_{L^{2}([0, T])}} \exp \left[-\frac{y^{2}}{2\|\phi\|_{L^{2}([0, T])}}\right] d y \\
\Psi(t, \omega)=\phi(t) \int_{\mathbb{R}} \frac{g(y)}{\sqrt{2 \pi}\|\phi\|_{L^{2}([0, T])}} \exp ^{\diamond}\left[-\frac{(y-Y(t))^{\diamond 2}}{2\|\phi\|_{L^{2}([0, T])}}\right] \diamond \frac{y-Y(t)}{\|\phi\|_{L^{2}([0, T])}} d y,
\end{gathered}
$$

$\diamond$ is Wick product and $\dot{\hat{W}}_{t}$ is the white noise of Wiener process $\hat{W}(t)$ under measure $Q$.

If we take $g(y)=\chi_{[K, \infty)}(y)$ and $Y(T)=W(T)$ which implies $\phi(t)=1, \alpha(t)=-u(t)$ where $u(t)$ is defined in equation (53) then $\Psi(t, \omega)$ is expressed as follows;

$$
\Psi(t, \omega)=\int_{K}^{\infty}(\sqrt{2 \pi})^{-1 / 2} T^{-1 / 2} \exp ^{\diamond}\left[-\frac{(y-W(t))^{\diamond 2}}{2 T}\right] \diamond \frac{y-W(t)}{T} d y,
$$


Also note that by using Clark-Ocone formula we can write the payoff of the digital function which is;

$$
\chi_{[K, \infty)}(W(T))=E_{Q}\left[\chi_{[K, \infty)}(W(T))\right]+\int_{0}^{T} E_{Q}\left[D_{t}\left\{\chi_{[K, \infty)}(W(T))\right\} \mid \mathcal{F}_{t}\right] d \hat{W}(t)
$$

Substituting equation (57) into the equation (56) and comparing the terms of the equation (58) with (56), we obtain the following result:

$$
E_{Q}\left[D_{t}\left\{\chi_{[K, \infty)}(W(T))\right\} \mid \mathcal{F}_{t}\right]=(2 \pi)^{-1 / 2} T^{-1 / 2} \int_{K}^{\infty} \exp ^{\diamond}\left[-\frac{(y-W(t))^{\diamond 2}}{2 T}\right] \diamond \frac{y-W(t)}{T} d y .
$$

By K. Aase et al (Lemma 3.8, page 362) [2], equation (59) equals to

$$
E_{Q}\left[D_{t}\left\{\chi_{[K, \infty)}(W(T))\right\} \mid \mathcal{F}_{t}\right]=(2 \pi)^{-1 / 2}(T-t)^{-1 / 2} \int_{K}^{\infty} \exp \left[-\frac{(y-W(t))^{2}}{2(T-t)}\right] \frac{y-W(t)}{T-t} d y .
$$

Therefore, in the replicating portfolio the number of the risky assets for hedging digital option should be

$$
\theta_{1}(t)=e^{-\rho(T-t)}(2 \pi(T-t))^{-1 / 2} \sigma^{-1}(t) S_{1}^{-1}(t) \int_{K}^{\infty} \exp \left[-\frac{(y-W(t))^{2}}{2(T-t)}\right] \frac{y-W(t)}{T-t} d y .
$$

\section{References}

[1] K. Aase, B. Øksendal, N. Privault and J. Ubøe, White noise generalizations of the ClarkHaussmann-Ocone theorem with application to mathematical finance, Finance and Stochastic 4 (2000), 465-496.

[2] K. Aase, B. Øksendal and J. Ubøe, Donsker delta function to compute hedging strategies, Potential Analysis 14 (2001), 351-374.

[3] G. Di Nunno, B. Øksendal and F. Proske, Malliavin Calculus for Lèvy Processes with Applications to Finance, Forthcoming book, to be published by Springer-Verlag.

[4] I. M. Gel'fand and N. Y. Vilenkin, Generalized Functions, Vol. 4, Academic Press, New York, 1964.

[5] T. Hida, Brownian Motion, Springer-Verlag, 1980.

[6] T. Hida, White noise analysis and its applications, Proc. Int. Math. Conf., ed. by Chen et al (1982), 43-48.

[7] T. Hida, H. Kuo, J. Potthoff and L. Streit, White Noise, An Infinite Dimensional Approach, Kluwer, 1993.

[8] H. Holden, B. Øksendal, J. Ubøe and T. S. Zhang, Stochastic Partial Differential Equations: A Modelling, White Noise Functioanal Approach, Birkhäuser, Boston, 1996.

[9] K. Itô, Multiple Wiener integral, Journal of Math. Soc. Japan 3 (1951), 157-169.

[10] I. Karatzas and D. Ocone, A generalized Clark representation formula, with application to optimal portfolios, Stoch. and Stoch. Rep. 34 (1991), 187-220.

[11] I. Karatzas and S. Shreve, Brownian Motion and Stochastic Claculus, Springer-Verlag, 1987.

[12] M. Loève, Probability Theory II, Springer-Verlag, 1978.

[13] D. Ocone, Malliavin calculus and stochastic integral representations of diffusion processes, Stochastics 12 (1984), 161-185. 